\title{
The white-dwarf cooling sequence of NGC 6791: a unique tool for stellar evolution
}

\author{
E. García-Berro ${ }^{1,2}$, S. Torres ${ }^{1,2}$ I. Renedo ${ }^{1,2}$, J. Camacho ${ }^{1,2}$, L. G. Althaus ${ }^{3,4}$, \\ A. H. Córsico ${ }^{3,4}$, M. Salaris ${ }^{5}$, and J. Isern ${ }^{2,6}$
}

\author{
${ }^{1}$ Departament de Física Aplicada, Universitat Politècnica de Catalunya, c/Esteve Terrades 5, 08860 Castelldefels, Spain \\ e-mail: garcia@fa.upc.edu \\ 2 Institute for Space Studies of Catalonia, c/Gran Capità 2-4, Edif. Nexus 104, 08034 Barcelona, Spain \\ ${ }^{3}$ Facultad de Ciencias Astronómicas y Geofísicas, Universidad Nacional de La Plata, Paseo del Bosque s/n, 1900 La Plata, Argentina \\ ${ }^{4}$ Instituto de Astrofísica de La Plata (CCT La Plata), CONICET, 1900 La Plata, Argentina \\ 5 Astrophysics Research Institute, Liverpool John Moores University, 12 Quays House, Birkenhead, CH41 1LD, UK \\ ${ }^{6}$ Institut de Ciències de l'Espai, CSIC, Campus UAB, Facultat de Ciències, Torre C-5, 08193 Bellaterra, Spain
}

Received 11 January 2011 / Accepted 15 July 2011

\begin{abstract}
Context. NGC 6791 is a well-studied, metal-rich open cluster that is so close to us that it can be imaged down to luminosities fainter than that of the termination of its white-dwarf cooling sequence, thus allowing for an in-depth study of its white dwarf population. Aims. White dwarfs carry important information about the history of the cluster. We use observations of the white-dwarf cooling sequence to constrain important properties of the cluster stellar population, such as the existence of a putative population of massive helium-core white dwarfs, and the properties of a large population of unresolved binary white dwarfs. We also investigate the use of white dwarfs to disclose the presence of cluster subpopulations with a different initial chemical composition, and we obtain an upper bound to the fraction of hydrogen-deficient white dwarfs.

Methods. We use a Monte Carlo simulator that employs up-to-date evolutionary cooling sequences for white dwarfs with hydrogenrich and hydrogen-deficient atmospheres, with carbon-oxygen and helium cores. The cooling sequences for carbon-oxygen cores account for the delays introduced by both ${ }^{22} \mathrm{Ne}$ sedimentation in the liquid phase and by carbon-oxygen phase separation upon crystallization.

Results. We do not find evidence for a substantial fraction of helium-core white dwarfs, and hence our results support the suggestion that the origin of the bright peak of the white-dwarf luminosity function can only be attributed to a population of unresolved binary white dwarfs. Moreover, our results indicate that if this hypothesis is at the origin of the bright peak, the number distribution of secondary masses of the population of unresolved binaries has to increase with increasing mass ratio between the secondary and primary components of the progenitor system. We also find that the observed cooling sequence appears to be able to constrain the presence of progenitor subpopulations with different chemical compositions and the fraction of hydrogen-deficient white dwarfs. Conclusions. Our simulations place interesting constraints on important characteristics of the stellar populations of NGC 6791. In particular, we find that the fraction of single helium-core white dwarfs must be smaller than $5 \%$, that a subpopulation of stars with zero metallicity must be $\lesssim 12 \%$, while if the adopted metallicity of the subpopulation is solar the upper limit is $\sim 8 \%$. Finally, we also find that the fraction of hydrogen-deficient white dwarfs in this particular cluster is surprinsingly small $(\lesssim 6 \%)$.
\end{abstract}

Key words. white dwarfs - stars: luminosity function, mass function - open clusters and associations: general open clusters and associations: individual: NGC 6791

\section{Introduction}

White dwarfs are the most common stellar evolutionary endpoint. Their evolution can be described as a very slow cooling process. Given that these fossil stars are abundant and longlived objects, they convey important information about the formation and evolution of all Galactic populations (Hansen \& Liebert 2003; Althaus et al. 2010a). In particular, white dwarfs can be employed as reliable cosmic clocks to infer the ages of a wide variety of stellar populations, such as the Galactic disk and halo (Winget et al. 1987; García-Berro et al. 1988a,b; Isern et al. 1998) and the system of globular and open clusters (Kalirai et al. 2001; Hansen et al. 2002). Additionally, they can be used to place constraints on exotic elementary particles (Isern et al. 1992; Córsico et al. 2001; Isern et al. 2008) or on alternative theories of gravitation (García-Berro et al. 1995; Benvenuto et al. 2004; García-Berro et al. 2011).
The use of white dwarfs as cosmochronometers relies on the accuracy of the theoretical cooling sequences. Recently, GarcíaBerro et al. (2010) have demonstrated that the slow-down of the white-dwarf cooling rate owing to the release of gravitational energy from ${ }^{22} \mathrm{Ne}$ sedimentation (Bravo et al. 1992; Bildsten \& Hall 2001; Deloye \& Bildsten 2002) and carbon-oxygen phase separation upon crystallization (García-Berro et al. 1988a; Segretain et al. 1994) is of fundamental importance to reconcile the age discrepancy of the very old, metal-rich open cluster NGC 6791. This raises the possibility of using the white-dwarf luminosity function of this cluster to constrain its fundamental properties.

NGC 6791 is one of the oldest Galactic open clusters - see, for instance, Bedin et al. (2005) and Kalirai et al. (2007), and references therein - and it is so close to us that it can be imaged down to very faint luminosities. A deep luminosity function of its well populated white dwarf sequence has been recently 
determined from HST observations (Bedin et al. 2008a) and displays a sharp cut-off at low luminosities, caused by the finite age of the cluster, plus a secondary peak at higher luminosities, most likely produced by a population of unresolved binary white dwarfs (Bedin et al. 2008b). These characteristics make this cluster a primary target to use the white-dwarf cooling sequence to constrain the presence of a population of massive heliumcore white dwarfs, the properties of the binary white-dwarf population, the hypothetical presence of cluster subpopulations of different metallicity, and the fraction of hydrogen-deficient (non-DA) white dwarfs. Here we address these questions by means of Monte-Carlo-based techniques aimed at producing synthetic color-magnitude diagrams and luminosity functions for NGC 6791 white dwarfs, which can be compared with the observational data.

Firstly, we investigate in detail the nature of the secondary peak of the white-dwarf luminosity function. This peak has been atributted to a population of unresolved binary white dwarfs (Bedin et al. 2008b) or to the existence of a population of single helium-core white dwarfs (Hansen 2005). This is a crucial question because if the first hypothesis is true, the amplitude of the secondary peak is such that leads to an unusual fraction of binary white dwarfs, thus challenging our understanding of the physical processes that rule the formation of binary white dwarfs, especially at high metallicities. Consequently, we also study other explanations for the existence of a secondary peak in the whitedwarf luminosity function, like the existence of a population of single helium-core white dwarfs. This explanation was first put forward by Hansen (2005) and Kalirai et al. (2007) and was later challenged, among others, by van Loon et al. (2008). However, as we will show below, this explanation results in a white-dwarf luminosity function that is at odds with the observed one, and hence the most likely explanation for the secondary bright peak is the population of unresolved binaries. Indeed, there is a strong reason to suspect that the bright peak of the white-dwarf luminosity function of NGC 6791 is caused by a population of unresolved binary white dwarfs, namely, that the two peaks of the white-dwarf luminosity function are separated by $0.75^{\mathrm{mag}}$. This is just exactly what it should be expected if the bright peak is caused by equal mass binaries. Hence, if this explanation for the bright peak of the white-dwarf luminosity function is true this, in turn, enables us to study the properties of the population of such binary white dwarfs. Specifically, we study how different distributions of secondary masses of the unresolved binary white dwarfs affect the color-magnitude diagram and the white-dwarf luminosity function.

Additionally, we test whether the white-dwarf luminosity function can provide an independent way to check for the existence of subpopulations within a stellar system. The presence of these subpopulations has been found in several Galactic globular clusters of which $\omega$ Cen is, perhaps, the most representative one (Calamida et al. 2009). The appearance of NGC 6791 color-magnitude diagram, and the lack of any significant chemical abundance spread (Carraro et al. 2006) points toward a very homogeneous stellar population. However, a recent paper by Twarog et al. (2011) raises the possibility that there could be a 1 Gyr age difference between inner and outer regions of the cluster. Nevertheless, within the field covered by the white dwarf photometry used in our analysis the age difference should be negigible. This is different from the case of individual Galactic globular clusters that host at least two distinct populations with approximately the same age and different abundance patterns of the C-N-O-Na elements - see Milone et al. (2010) for a recent brief review. Taking advantage of the well-populated cooling sequence in the observed color-magnitude diagram, we test whether modeling the cluster white dwarf color-magnitude alone can exclude the presence of subpopulations generated by progenitors with a metallicity different from the one measured spectroscopically.

As a final study, we use the luminosity function of NGC 6791 (Bedin et al. 2008a), and the fact that white dwarfs with hydrogen-rich atmospheres (of the DA type) and non-DA white dwarfs cool at a different rate, to place constraints on the fraction of these objects. This is an important point because Kalirai et al. (2005) have found that in the young open star cluster NGC 2099 there is a dearth of non-DA white dwarfs. These authors attributed the lack of non-DA objects to the fact that possibly the hot, high-mass cluster white dwarfs - white dwarfs in this cluster are estimated to be more massive than field objects in the same temperature range - do not develop sufficiently extended helium convection zones to allow helium to be brought to the surface and turn a hydrogen-rich white dwarf into a helium-rich one. Studying the fraction of non-DA white dwarfs in a different open cluster could provide additional insight into this question. Moreover, Kalirai et al. (2007) analyzed a sample of $\sim 15$ white dwarfs in NGC 6791, and although the sample was far from being complete, all them were of the DA type.

The paper is organized as follows. Section 2 summarizes the main ingredients of our Monte Carlo code plus the other basic assumptions and procedures necessary to evaluate the characteristics of the white dwarf population for the different simulations presented here. Specifically, we discuss the most important ingredients used to construct reliable color-magnitude diagrams and the corresponding white-dwarf luminosity functions. Section 3 presents the main results of our simulations for all points already mentioned in this section. Finally, Sect. 4 closes the paper with a summary of our conclusions.

\section{Modeling NGC 6791}

\subsection{The Monte-Carlo simulator}

The photometric properties of NGC 6791 were simulated using a Monte-Carlo technique. Our Monte Carlo simulator has been previously employed to model the Galactic disk and halo field white dwarf populations (García-Berro et al. 1999; Torres et al. 2002; García-Berro et al. 2004), with excellent results. We briefly describe here the most important ingredients of our simulator. Synthetic main-sequence stars are randomly drawn according to a Salpeter-like initial mass function that in the mass range relevant to NGC 6791 white dwarf progenitors $\left(M>1.0 M_{\odot}\right)$ is essentially identical to the "universal" initial mass function of Kroupa (2001), and a burst of star formation starting 8 Gyr ago, lasting $0.1 \mathrm{Gyr}$ (the exact value is not critical for our analysis). If not otherwise stated, we account for a population of unresolved detached binary white dwarfs adopting a fraction $f_{\text {bin }}=0.54$ of binary systems in the main sequence, which leads to a fraction of unresolved white dwarf binaries of $36 \%$. In our fiducial model we use the distribution of secondary masses previously employed by Bedin et al. (2008b), but other distributions were also used (see Sect. 3.2) to constrain the properties of the binary population. We employ main-sequence lifetimes from the calculations by Weiss \& Ferguson (2009) for $Z=0.04, Y=0.325$ models, which correspond to $[\mathrm{Fe} / \mathrm{H}] \sim 0.4$, a metallicity consistent with the recent spectroscopic determinations of Carraro et al. (2006), Gratton et al. (2006) and Origlia et al. (2006). For the white dwarf initial-final mass relationship we used that of Ferrario et al. (2005), which is appropriate for metal-rich stars, 
although our results are fairly insensitive to the precise choice of this function. For instance, when the up-to-date semiempirical initial-final mass function of Catalán et al. (2008) is adopted, the results are almost indistinguishable from those obtained with the former relationship.

Given the cluster age, the time at which each synthetic star was born and its associated main sequence lifetime, we kept track of the stars able to evolve to the white dwarf stage, and we interpolated their colors and luminosities using the theoretical cooling sequences described in the following subsection. For unresolved binary systems we performed the same calculation for the secondary and we added the fluxes and computed the corresponding colors. The photometric errors were drawn according to Gaussian distributions, using the Box-Müller algorithm as described in Press et al. (1986). The standard photometric errors in magnitude and color were assumed to increase linearly with the magnitude following Bedin et al. (2005, 2008a). Finally, we added the distance modulus of NGC 6791, $(m-M)_{F 606 W}=$ 13.44 , and its color excess $E(F 606 W-F 814 W)=0.14$ (Bedin et al. 2008a,b) to obtain a synthetic white dwarf color-magnitude diagram, and from this we computed the corresponding whitedwarf luminosity function. The distance modulus adopted here agrees with the recent estimate of Grundahl et al. (2008), which is based on a cluster eclipsing binary system.

\subsection{The cooling sequences}

The cooling sequences adopted in this work were those previously employed by García-Berro et al. (2010), which were extensively discussed in Althaus et al. (2010b). These cooling sequences are appropriate for white dwarfs with hydrogen-rich atmospheres, and were computed from stellar models with the metallicity of NGC 6791. In summary, these sequences were obtained from star models with stellar masses at the ZAMS ranging from 1 to $3 M_{\odot}$ and were followed through the thermally pulsing and mass-loss phases on the AGB to the white dwarf stage. The resulting white dwarf masses were $0.5249,0.5701,0.593$, $0.6096,0.6323,0.6598$ and $0.7051 M_{\odot}$. Along the white dwarf cooling track the calculations include the release of gravitational energy resulting from ${ }^{22} \mathrm{Ne}$ diffusion in the liquid phase and from phase separation of carbon and oxygen upon crystallization. The energy contributions of these two physical separation processes were computed by closely following the prescriptions of Isern et al. (1997) and Isern et al. (2000). We stress here that this was made in a self-consistent way, because the energy release is locally coupled to the equations of stellar evolution. Finally, we mention that our calculations rely on realistic boundary conditions for cool white dwarfs, as given by non-gray model atmospheres (Rohrmann et al. 2010). For non-DA white dwarfs a new set of cooling sequences was computed from the same set of progenitors, using the same physical inputs as adopted in Althaus et al. (2010b), the only difference being the chemical composition of the atmosphere, for which we adopted pure helium. Details of these calculations will be presented elsewhere. The bolometric corrections and color transformations for this set of cooling sequences were those of Bergeron et al. (1995).

\section{Results}

\subsection{A population of single helium-core white dwarfs?}

As mentioned, the bright peak of the white-dwarf luminosity function of NGC 6791 has been attributed either to a huge population of binary white dwarfs (Bedin 2008b) or to the existence of a population of single helium-core white dwarfs (Hansen 2005; Kalirai 2007). This would indicate that at the very high metallicity of this cluster mass-loss at the tip of the red giant branch would be largely enhanced. This possibility has been recently investigated, among others, by Meng et al. (2008), who found - on the basis of specific assumptions about the minimum envelope mass of red or asymptotic giant-branch stars - that for $Z \gtrsim 0.02$ helium white dwarfs are likely the result of the evolution of stars with masses smaller than 1.0 $M_{\odot}$. However, Prada Moroni \& Straniero (2009) have demonstrated that when the star loses the envelope and departs from the red giant branch with a core mass slightly smaller than that required for helium ignition, a non-negligible possibility of a late helium ignition exists, and low-mass carbon-oxygen white dwarfs, rather than helium-core white dwarfs, are produced. Thus, more studies to constrain a hypothetical population of single helium-core white dwarfs are needed.

To this end we have proceeded as follows. At the metallicity of NGC 6791, the helium-core mass at the helium flash is $\sim 0.45 M_{\odot}$, practically constant with initial mass, up to the transition to non-degenerate cores (Weiss \& Ferguson 2009). Also, adopting an age of $8 \mathrm{Gyr}$, the mass at the turn-off is $\sim 1.15 M_{\odot}$ and the maximum mass of progenitors that could have made a helium-core white dwarf is $\sim 1.8 M_{\odot}$. This means that the range of masses of possible progenitors of single white dwarfs with helium cores is between $\sim 1.15$ and $\sim 1.8 M_{\odot}$. Accordingly, we draw a pseudo-random number for the mass of the progenitor using our initial mass function and we consider that a fraction $f_{\mathrm{He}}$ of stars between 1.15 and $2.0 M_{\odot}$ have lost the envelope near the tip of the red giant branch and produce helium-core white dwarfs with masses between 0.2 and $0.5 M_{\odot}$. The adopted cooling sequences for helium-core white dwarfs are those of Althaus et al. (2009).

The results of our simulations are displayed in the top panels of Fig. 1, where we show the synthetic color-magnitude diagram and white-dwarf luminosity function of the cluster for the case in which we adopt $f_{\mathrm{He}}=0.3$ and $f_{\text {bin }}=0$. Clearly, this scenario is unable to reproduce the bright peak of the white-dwarf luminosity function and the corresponding clump in the color-magnitude diagram. Indeed, most helium-core white dwarfs are located in the same region of the color-magnitude diagram where regular carbon-oxygen white dwarfs are placed, the only difference is the position of the cut-off. In fact, helium-core white dwarfs pile-up at higher luminosities than carbon-oxygen ones, as expected, but at luminosities slightly fainter than that of the observational bright peak. The net result of this is that the population of helium-core white dwarfs partially overlaps with the peak produced by normal carbon-oxygen white dwarfs and, consequently, the faint peak of the white-dwarf luminosity function broadens.

Now that we have established that single helium-core white dwarfs cannot explain the bright peak of the white-dwarf luminosity function, a natural question arises. Namely, which is the maximum fraction of these white dwarfs compatible with observations? To answer this question we performed additional simulations using our fiducial model, in which a population of unresolved detached binaries is adopted - see Sect. 2.1 - and we added a small fraction $f_{\mathrm{He}}$ of helium-core white dwarfs, keeping the fraction of binary white dwarfs of the cluster constant. For illustrative purposes, the bottom panels of Fig. 1 show the case in which $f_{\mathrm{He}}=0.3$ and $f_{\text {bin }}=0.54$ are adopted. A $\chi^{2}$ test shows that the maximum fraction of single massive helium-core white dwarfs allowed by the observations is $f_{\mathrm{He}}=0.05$. 

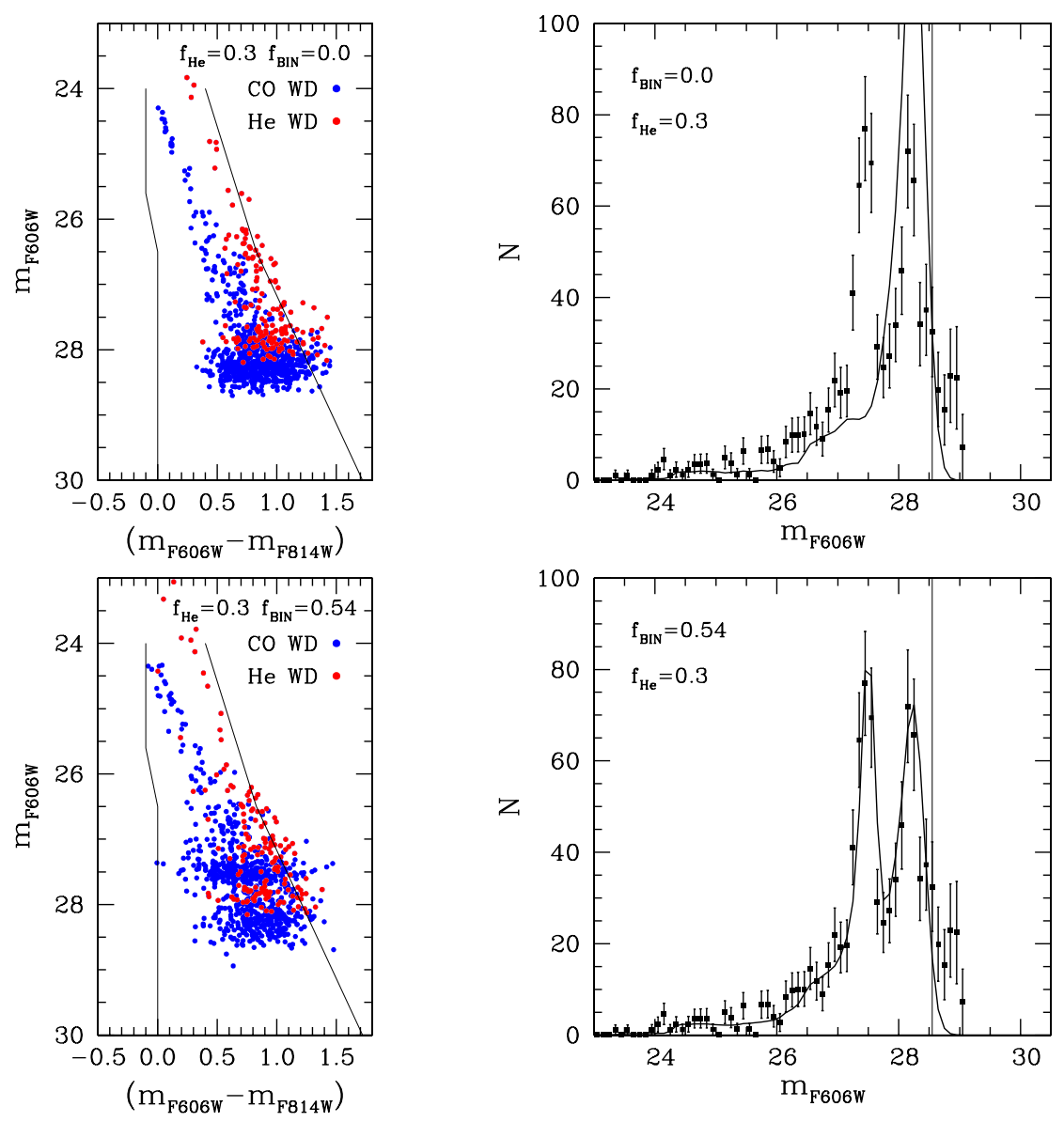

Fig. 1. Color-magnitude diagrams (left panels) of the synthetic population of carbon-oxygen white dwarfs (blue symbols) and of helium-core white dwarfs (red symbols) and the corresponding white-dwarf luminosity functions (right panels). The observational selection area in the colormagnitude diagram of Bedin et al. (2005) is also shown using thin lines. These boundaries in the color-magnitude exclude low-mass helium-core white dwarfs. The observational white-dwarf luminosity function of Bedin et al. (2008b), which was corrected for incompleteness, is shown using black squares. Each theoretical luminosity function corresponds to an average of $10^{4}$ Monte Carlo realizations. The vertical thin line marks the magnitude limit $\simeq 28.5^{\mathrm{mag}}$ above which the completeness level of the photometry falls below $50 \%$. The top panels correspond to the case in which $f_{\mathrm{He}}=0.3$ and $f_{\text {bin }}=0.0$ are adopted, while for the bottom ones we adopted $f_{\mathrm{He}}=0.3$ and $f_{\text {bin }}=0.54$. See the online edition of the journal for a color version of this figure.

\subsection{The properties of the binary population}

If the bright peak of the white-dwarf luminosity function of NGC 6791 can only be explained by a population of unresolved binary white dwarfs, their properties can be constrained using the white-dwarf luminosity function. There are other clusters (either open or globular) for which we have observational white-dwarf luminosity functions - M67 (Richer et al. 1998), NGC 2099 (Kalirai et al. 2001), NGC 188 (Andreuzzi et al. 2002), M4 (Hansen et al. 2004), and NGC 6397 (Hansen et al. 2007; Richer et al. 2008) - and none of them shows a secondary peak in the white-dwarf luminosity function with the characteristics of that of NGC 6791. Perhaps the best studied of these clusters is NGC 6397. This cluster has been imaged down to very faint luminosities allowing Hansen et al. (2007) and Richer et al. (2008) to obtain a reliable white-dwarf luminosity function. The white-dwarf luminosity function of this very old - about 12 Gyr (di Criscenzo et al. 2010) - and metal-poor $([\mathrm{Fe} / \mathrm{H}] \simeq-2.03 \pm 0.05)$ globular cluster does not show any evidence of a large population of binary white dwarfs. There is another open cluster for which a large number of binaries has been found, M67 (Richer et al. 1998). The age of this cluster is $4.0 \mathrm{Gyr}$, and its metallicity is almost solar, $[\mathrm{Fe} / \mathrm{H}] \simeq-0.04$, but unfortunately the small number of white dwarfs with reliable photometry does not allow to perform a thorough study of its population of binary white dwarfs. All in all, NGC 6791 offers us the unique possibility to study the properties of the population of binaries in an open, very old and metal-rich cluster. In particular, we study how the white-dwarf luminosity function allows to constrain the distribution of secondary masses. However, before performing our analysis we note that blending may have the same effect than true unresolved binaries, although in the case of an open cluster, it is expected to be less frequent, owing to the lower density of stars. To quantify this, we distributed 900 synthetic white dwarfs (the observed number of stars) in the field of view of the HST CCD $(4052 \times 4052$ pixels $)$ and we evaluated the probability of chance superposition. We found that this probability is $\sim 0.8 \%$ if the distance necessary to resolve two stars is $\sim 10$ pixels. Thus, for the case of NGC 6791 this possibility is quite unlikely, and the unresolved binary white dwarfs are most probably real systems.

We used four different models for the distribution of secondary masses in the progenitor bynary system, under the assumption - the same as in Bedin et al. (2008b) - that binary white dwarfs are produced by a primordial binary system. Our first distribution is that already used by Bedin et al. (2008b), 
E. García-Berro et al.: The white dwarf cooling sequence of NGC 6791
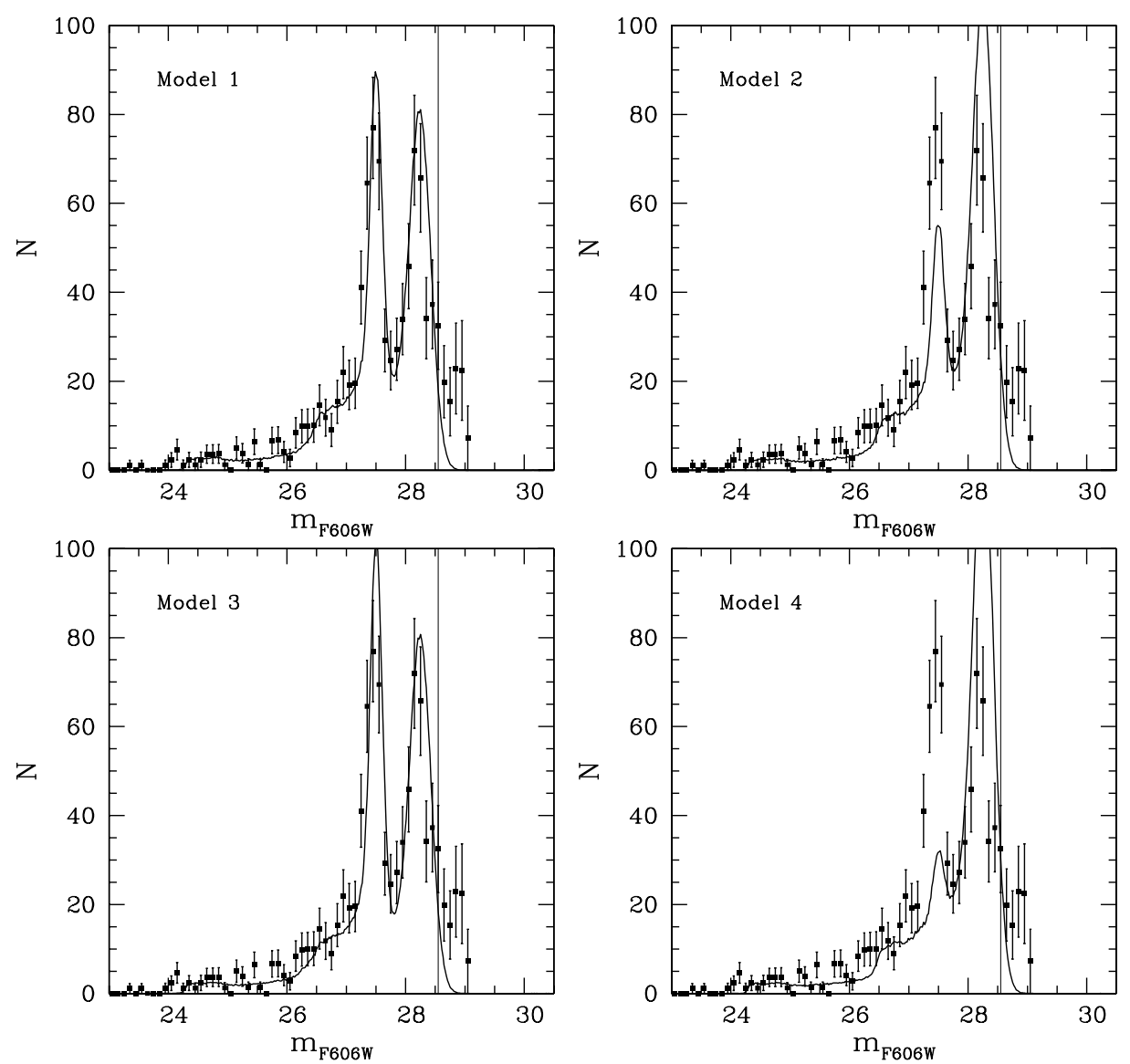

Fig. 2. White-dwarf luminosity functions for several distributions of secondary masses of the progenitor binary system, see text for details.

$n(q)=0.0$ for $q<0.5$ and $n(q)=1.0$ otherwise, where $q=M_{2} / M_{1}$, being $M_{1}$ and $M_{2}$ the mass of the primary and of the secondary, respectively. We refer to this distribution as model 1. In model 2 we assume $n(q)=1.0$, independently of the mass ratio. For model 3 we adopted $n(q) \propto q$. Finally, for our last set of simulations, corresponding to model 4 , we adopted $n(q) \propto q^{-1}$.

We display the results of this set of simulations in Fig. 2. Evidently, the corresponding white-dwarf luminosity functions show dramatic differences. The distribution of secondary masses of Bedin et al. (2008b), top left panel, perfectly matches the observational white-dwarf luminosity function of NGC 6791. When model 2 is adopted, the secondary peak of the simulated luminosity function does not match the observational data, and the amplitude of the faintest peak is very much increased. It might be argued that this incongruence could be fixed by simply changing the fraction of binary white dwarfs, and indeed this could be done, but then one would need a present total percentage of binary stars well above $60 \%$, which is probably unrealistic. Thus, we conclude that a flat distribution of secondary masses can be discarded. When the third distribution of secondary masses is used, we obtain a good fit to the observational data, although the quality of the fit is not as good as that of model 1. This is not surprising, since both distributions of secondary masses increase for increasing values of $q$. Finally, when model 4 is employed, the simulated white-dwarf luminosity function is totally incompatible with the observational data. The same arguments used when discussing the flat distribution of secondary masses apply here, and thus we can safely discard this distribution. We conclude that most likely only distributions of secondary masses that increase as the mass ratio of the two components of the binary increases are compatible with the existing observational data for NGC 6791.

\subsection{Identification of cluster subpopulations: a test case}

Our understanding of Galactic open and globular clusters has dramatically changed in recent years owing to the wealth of precise photometric data. This has allowed us to unveil the presence of multiple main sequences or subgiant branches - see, for instance, Piotto et al. (2007) and Milone et al. (2008) - in several globular clusters. Nowadays there is a handful of Galactic globular clusters in which the presence of several subpopulations is notorious. The best known of these clusters is $\omega$ Cen, for which four different metallicity regimes have been so far identified (Calamida et al. 2009). However, $\omega$ Cen is not the only example. For instance, Piotto et al. (2007) have convincingly shown that the main sequence of the globular cluster NGC 2808 contains three distinct subpopulations, while Milone et al. (2008) have demonstrated that NGC 1851 hosts a double subgiant branch, and di Criscienzo et al. (2010) have shown that NGC 6397 may contain a large number of second-generation stars. Thus, the presence of multiple populations in globular clusters is not an infrequent phenomenon.

To the best of our knowledge there is no evidence for the occurrence of this phenomenon in old open clusters. NGC 6791 is particularly well suited to study this possibility. Firstly, it is very old, a characteristic shared with globular clusters. Secondly, NGC 6791 is extremely metal-rich. The origin of this metallicity enhancement is still unknown, but arguably could be due to the existence of a previous generation of metalpoor stars. Nevertheless, we stress that in other clusters these 

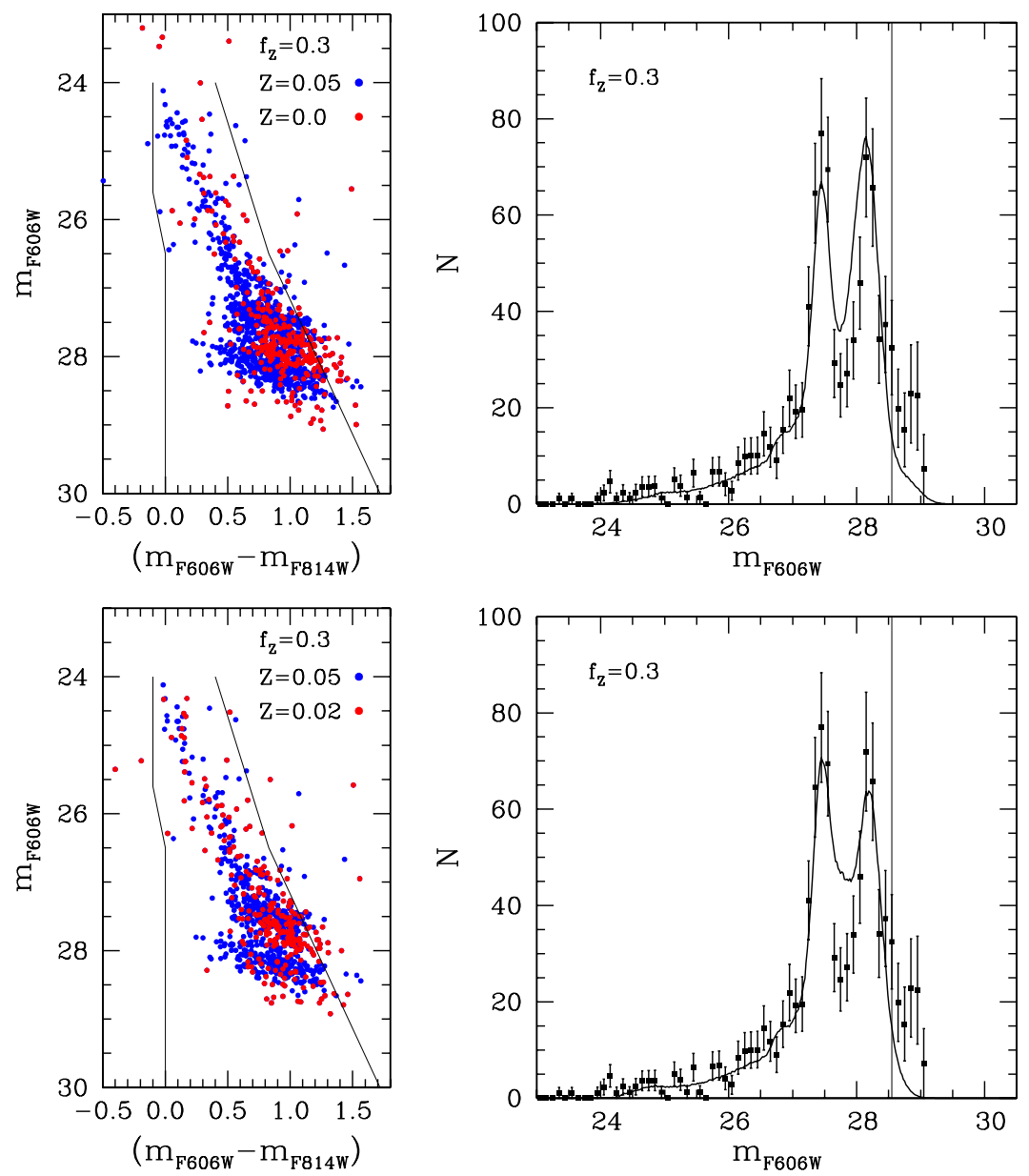

Fig. 3. Color-color diagrams (left panels) of the simulated subpopulations of white dwarfs with metal-rich progenitors (blue circles) and metal-poor progenitors (red circles), for two metallicites of the subpopulations, $Z=0.0$ (top panel) and $Z=0.02$ (bottom panel). In both cases we show the results for a fraction of the subpopulation $f_{Z}=0.3$. The resulting whitedwarf luminosity functions (solid lines) are compared to the observational one (squares) in the right panels. See the online edition of the journal for a color version of this plot.

subpopulations are observed as multiple values of $[\mathrm{Fe} / \mathrm{H}]$ (and also helium), but a recent spectroscopical analysis of NGC 6791 by Origlia et al. (2006) shows that there is no spread in $[\mathrm{Fe} / \mathrm{H}]$, and no spread in carbon or oxygen, which are two of the elements involved in the related subpopulations. Also, the well-defined red giant branch argues against this hypothesis. However, there are other clusters, of which NGC 6397 is the best example, which show remarkably clean color-magnitude diagrams with very tight main sequences and compact blue horizontal branches - that is, with no obvious photometric signs of multiple populations - for which subpopulations have already been identified (Lind et al. 2010). Finally, nobody has yet explored the possibility of using the white-dwarf luminosity function to put constraints on multiple subpopulations in clusters, although Prada Moroni \& Straniero (2007) already noted that the white dwarf isochrones are considerably affected by metallicity variations. Thus, it is interesting to carry out this sensitivity study taking advantage of the well-populated white dwarf colormagnitude diagram, to test whether just modeling the white dwarf population can exclude the presence of subpopulations generated by progenitors with a metallicity different from the one measured spectroscopically.

To perform this study, we first considered varying fractions $f_{Z}$ of an extreme subpopulation with zero metallicity. The pre-white dwarf lifetimes were taken from Marigo et al. (2001), whilst the carbon-oxygen stratification in the white dwarf models was kept unchanged, given the small effect of the progenitor initial chemical composition on the final carbon-oxygen profiles and cooling times at fixed white dwarf mass - see, for instance, Salaris et al. (2010) and Renedo et al. (2010). We also neglected the delay introduced by ${ }^{22} \mathrm{Ne}$ sedimentation, to account for the negiglible abundance of this element in $Z=$ 0 models. The reason for this is the following. ${ }^{22} \mathrm{Ne}$ is produced during the helium burning phase by the chain of reactions ${ }^{14} \mathrm{~N}(\alpha, \gamma){ }^{18} \mathrm{~F}\left(\beta^{+}\right){ }^{18} \mathrm{O}(\alpha, \gamma){ }^{22} \mathrm{Ne}$. The net effect is to transform essentially all ${ }^{14} \mathrm{~N}$ into ${ }^{22} \mathrm{Ne}$. In the extreme case of $Z=0$ stars some ${ }^{14} \mathrm{~N}$ is produced when the $\mathrm{CNO}$ cycle is activated by the ${ }^{12} \mathrm{C}$ produced by $3 \alpha$ reactions ignited by the high temperatures during the main sequence phase of metal free stars. However, its abundance mass fraction is $\lesssim 10^{-9}$ (Weiss et al. 2000).

The results of our simulations are shown in Fig. 3. We begin by discussing the color-magnitude diagram shown in upper left panel. Obviously, white dwarfs resulting from metal-poor progenitors detach from the bulk of the population, and several of these synthetic white dwarfs can be found below the welldefined cut-off of the observed cooling sequence, as expected, because the lack of ${ }^{22} \mathrm{Ne}$ causes a faster cooling. The upper right panel of this figure shows the corresponding luminosity function. The overall agreement with the observed luminosity function is poor, especially in the region between the two peaks. An increase of the binary fraction to reproduce the bright peak better would not improve the modeling of the region between the peaks. The natural question to address is then which is the maximum fraction of metal-poor white dwarf progenitors that can be accommodated within the observational white-dwarf luminosity function? To this purpose we have computed synthetic white dwarf samples with decreasing fractions of metal-poor progenitors, and we ran a $\chi^{2}$ test. We found that the maximum allowed fraction of metal-poor progenitors is $f_{Z}=0.12$. Obviously, the assumption that this subpopulation has zero metallicity is 

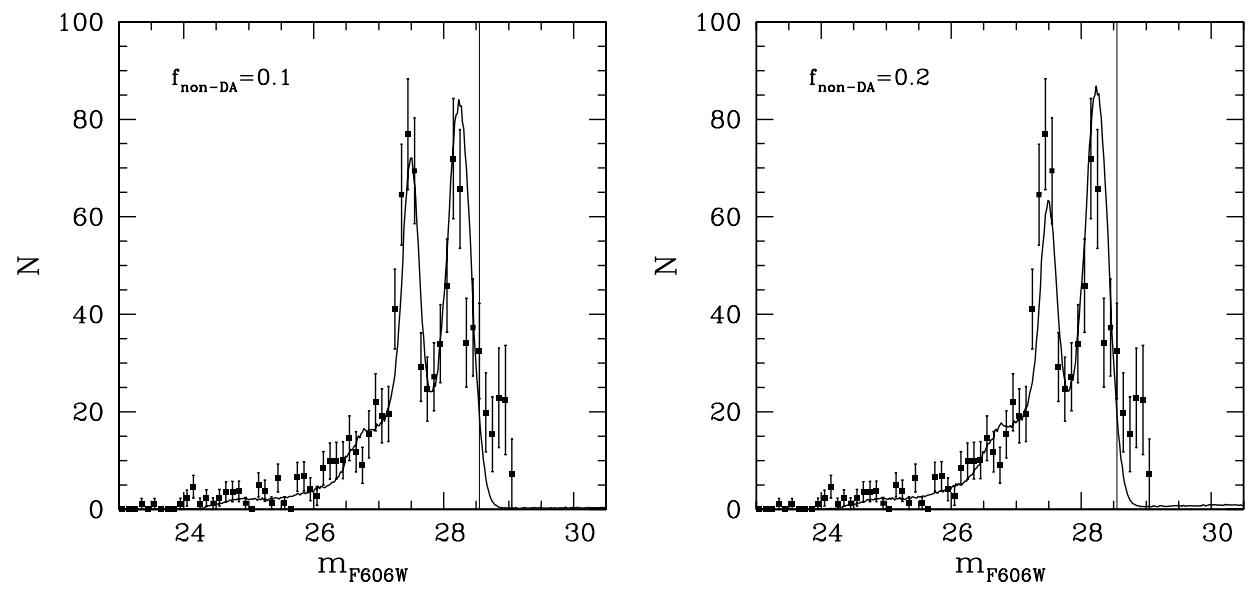

Fig. 4. Simulated luminosity functions for different fractions of non-DA white dwarfs, as shown in the corresponding panel.

probably to extreme. Consequently, we repeated the same calculation for a subpopulation of solar metallicity - see the bottom panels of Fig. 3. In this case the maximum fraction of solarmetallicity progenitors is $f_{Z}=0.08$.

\subsection{The fraction of non-DA white dwarfs}

We now focus on the possibility of determining the fraction of non-DA white dwarfs in NGC 6791. The spectral evolution of white dwarf atmospheres is still a controversial question, and although the ratio of white dwarfs with pure hydrogen atmosphere versus white dwarfs with hydrogen-deficient atmospheres is known for the local field, very few determinations exist for open and globular clusters. Moreover, although for the field white dwarf population the canonical percentage is around $80 \%$, observations show that this ratio depends on the effective temperature - see, for instance, Tremblay \& Bergeron (2008) and references therein. However, the only reliable determinations for open clusters are those of Kalirai et al. (2005) for the rich, young cluster NGC 2099, and Rubin et al. (2008) for NGC 1039. Kalirai et al. (2005) found a clear deficit of non-DA white dwarfs in NGC 2099, whereas Rubin et al. (2008) found that the fraction of non-DA white dwarfs in the open cluster NGC 1039 is $\sim 10 \%$, at most. Clearly, investigating the DA to non-DA ratio in another open cluster is therefore of greatest interest.

We addressed this question by simulating the cluster population of white dwarfs with an increasing fraction of non-DA stars. The non-DA fractions adopted here are $f_{\text {non-DA }}=0.0,0.1$, 0.2 and 0.4 , respectively. For the sake of conciseness, we only show the results for $f_{\text {non-DA }}=0.1-$ left panel - and $0.2-$ right panel. As shown in Fig. 4 the white-dwarf luminosity function is sensitive to the ratio of non-DA to DA white dwarfs. We find that when the fraction of non-DA white dwarfs is increased, the agreement with the observational white-dwarf luminosity function rapidly degrades. To be precise, when the fraction of nonDA white dwarfs is $f_{\text {non-DA }}=0.1$, the agreement is quite poor, and when the fraction of non-DA white dwarfs is that of field white dwarfs, $f_{\text {non-DA }}=0.2$, the quality of the fit to the observational white-dwarf luminosity function is unacceptable. This is because for the age of NGC 6791 non-DA and DA white dwarfs pile-up at similar luminosities. As a consequence, adding single non-DA white dwarfs lowers the height of the bright peak compared to the faint one.

To quantify which is the maximum fraction of non-DA white dwarfs that can be accommodated within the observational errors is, we conducted a $\chi^{2}$ test, and we found that for fractions of non-DA white dwarfs larger than $\sim 0.1$ the probability rapidly drops below $\sim 0.7$, whereas for $f_{\text {non-DA }}=0.0$ the probability is $\sim 0.9$. Consequently, the fraction of non-DA white dwarfs in NGC 6791 can roughly be at most half the value found for field white dwarfs. This result qualitatively agrees with the findings of Kalirai et al. (2005), who find that for NGC 2099 this deficit of non-DA white dwarfs is even higher. As a matter of fact, Kalirai et al. (2005) found that for this cluster all white dwarfs in their sample were of the DA type. Our results also point in the same direction, although a fraction of $\sim 5 \%$ is still compatible with the observed white-dwarf luminosity function of NGC 6791, in agreement with the findings of Kalirai et al. (2007).

Finally, we considered also the possibility that the fraction of non-DA white dwarfs changes with the effective temperature, which occurs with field white dwarfs. In particular, we assumed that for effective temperatures higher than $6000 \mathrm{~K}$, the fraction of non-DA white dwarfs is $f_{\text {non-DA }}=0.2$ and for temperatures ranging from $5000 \mathrm{~K}$ to $6000 \mathrm{~K}, f_{\text {non-DA }}=0.0$, as suggested by observations of low-luminosity field white dwarfs. In this case we find that the simulated white-dwarf luminosity function and color-magnitude diagram are very similar to those in which $f_{\text {non-DA }}=0.0$, and thus agree very well with the observational luminosity function of NGC 6791. Nevertheless, it is worth noting that using this prescription, the fraction of non-DA white dwarfs expected in the cluster would be about $6 \%$. Thus, on the basis of these simulations it cannot be discarded that this cluster could have originally produced a large percentage of non-DA white dwarfs, but at the present age of the cluster, most of them could have been transformed into DA white dwarfs as a result of accretion episodes.

These conclusions clearly depend on the assumed fraction of binary white dwarfs that populate the cluster. The fraction of white dwarf binaries necessary to explain the bright peak of the luminosity function (in absence of non-DA objects) requires that about $54 \%$ of the objects in NGC 6791 be binaries. An increased white dwarf binary percentage $(75 \%)$ can in principle accommodate a higher percentage $(20 \%)$ of non-DA objects by increasing the relative height of the bright peak in the synthetic sample, compared to the faint one, but it seems unrealistic to accept such a large percentage of cluster binaries, hence non-DA white dwarfs.

\section{Conclusions}

In this paper we have investigated several important properties of the stellar population hosted by the very old ( $8 \mathrm{Gyr}$ ), metal-rich $([\mathrm{Fe} / \mathrm{H}] \simeq 0.4)$ open cluster NGC 6791. This cluster has been imaged below the luminosity of the termination of 
its white dwarf cooling sequence (Bedin et al. 2005, 2008a). The resulting white-dwarf luminosity function enables us not only to determine the cluster age (García-Berro et al. 2010), but other important properties as well. Among these, we mention the properties of the population of unresolved binary white dwarfs, the existence of cluster subpopulations, and the fraction of non-DA white dwarfs.

The origin of the bright peak of the white-dwarf luminosity function was investigated, exploring in detail the alternative massive helium-core white dwarf scenario. Our conclusion is that this peak cannot be attributed to a population of single helium-core white dwarfs. The more realistic possibility left to explain this feature is a population of unresolved binary white dwarfs. This huge population of unresolved binary white dwarfs has allowed us to study the properties of the parent population. To this purpose, we studied the properties of the distribution of secondary masses in the binary progenitor system and its effects in the white-dwarf luminosity function. Specifically, we tested four different distributions of secondary masses and we found that only those distributions that are monotonically increasing with the mass ratio are consistent with the observational data. Additionally, as a test case, we verified the ability of the whitedwarf luminosity function to assess the existence of subpopulations within a stellar system. We have found that the presence of a $Z=0$ subpopulation is inconsistent with the white-dwarf luminosity function, the maximum fraction allowed by the data being $12 \%$. If the metallicity of the subpopulation is solar, this fraction is $8 \%$.

Finally, we found that the fraction of non-DA white dwarfs in this cluster is unusually small, on the order of $6 \%$ at most, and much smaller than the corresponding one for field white dwarfs, which is $\sim 20 \%$. This shortage of non-DA white dwarfs is a characteristic shared with another open cluster, NGC 2099. However, the deficit of non-DA white dwarfs is even higher in the case of NGC 2099, given that for this cluster recent exhaustive observations have found no single white dwarf of the non-DA type (Kalirai et al. 2005).

Acknowledgements. This research was supported by AGAUR, by MCINN grants AYA2008-04211-C02-01 and AYA08-1839/ESP, by the European Union FEDER funds, by the ESF EUROGENESIS project (grant EUI2009-04167), by AGENCIA: Programa de Modernización Tecnológica BID 1728/OC-AR, and by PIP 2008-00940 from CONICET. L.G.A. also acknowledges a PIV grant of the AGAUR of the Generalitat de Catalunya. We also thank our referee, O. Straniero, for useful comments and suggestions.

\section{References}

Althaus, L. G., Panei, J. A., Romero, A. D., et al. 2009, A\&A, 502, 207 Althaus, L. G., Córsico, A. H., Isern, J., \& García-Berro, E. 2010a, A\&AR, 18, 471

Althaus, L. G., García-Berro, E., Renedo, et al. 2010b, ApJ, 719, 612 Andreuzzi, G., Richer, H. B., Limongi, M., \& Bolte M. 2002, A\&A, 390, 961 Bedin, L. R., Salaris, M., Piotto, G., et al. 2005, ApJ, 624, L45 Bedin, L. R., King, I. R., Anderson, J., et al. 2008a, ApJ, 678, 1279 Bedin, L. R., Salaris, M., Piotto, G., et al. 2008b, ApJ, 679, L29 Benvenuto, O. G., García-Berro, E., \& Isern, J. 2004, Phys. Rev. D, 69, 082002
Bergeron, P., Saumon, D., \& Wesemael, F. 1995, ApJ, 443, 764

Bildsten, L., \& Hall, D. M. 2001, ApJ, 549, L219

Bravo, E., Isern, J., Canal, R., et al. 1992, A\&A, 257, 534

Calamida, A., Bono, G., Stetson, P. B., et al. 2009, ApJ, 706, 1277

Carraro, G., Villanova, S., Demarque, P., et al. 2006, ApJ, 643, 1151

Catalán, S., Isern, J., García-Berro, E., \& Ribas, I. 2008, MNRAS, 387, 1693

Córsico, A. H., Benvenuto, O. G., Althaus, L. G., Isern, J., \& García-Berro, E. 2001, New A, 6, 197

Deloye, C. J., \& Bildsten, L. 2002, ApJ, 580, 1077

di Criscienzo, M., D’Antona, F., \& Ventura, P. 2010, A\&A, 511, A70

Ferrario, L., Wickramasinghe, D., Liebert, J., \& Williams, K. A. 2005, MNRAS, 361,1131

García-Berro, E., Hernanz, M., Mochkovitch, R., \& Isern, J. 1988a, A\&A, 193, 141

García-Berro, E., Hernanz, M., Isern, J., \& Mochkovitch, R. 1988b, Nature, 333, 642

García-Berro, E., Hernanz, M., Isern, J., \& Mochkovitch, R. 1995, MNRAS, 277, 801

García-Berro, E., Torres, S., Isern, J., \& Burkert, A. 1999, MNRAS, 302, 173

García-Berro, E., Torres, S., Isern, J., \& Burkert, A. 2004, A\&A, 418, 53

García-Berro, E., Torres, S., Althaus, L. G., et al. 2010, Nature, 465, 194

García-Berro, E., Lorén-Aguilar, P., Torres, S., Althaus, L. G., \& Isern, J. 2011, J. Cosmology Astropart. Phys., 5, 21

Gratton, R., Bragaglia, A., Carretta, E., \& Tosi, M. 2006, ApJ, 642, 462

Grundahl, F., Clausen J. V., Hardis, S., \& Frandsen, S. 2008, A\&A, 492, 171

Hansen, B. M. S. 2005, ApJ, 635, 522

Hansen, B. M. S., \& Liebert, J. 2003, ARA\&A, 41, 465

Hansen, B. M. S., Brewer, J., Fahlman, G. G., et al. 2002, ApJ, 574, L155

Hansen, B. M. S., Richer, H. B., Fahlman, G. G., et al. 2004, ApJS, 155, 551

Isern, J., Hernanz, M., \& García-Berro, E. 1992, ApJ, 392, L23

Isern, J., García-Berro, E., Hernanz, M., Mochkovitch, R., \& Torres, S. 1998, ApJ, 503, 239

Isern, J., García-Berro, E., Hernanz, M., \& Chabrier, G. 2000, ApJ, 528, 397

Isern, J., García-Berro, E., Torres, S., \& Catalán, S. 2008, ApJ, 682, L109

Kalirai, J. S., Ventura, P., Richer, H. B., et al. 2001, AJ, 122, 3239

Kalirai, J. S., Richer, H. B., Hansen, B. M. S., Reitzel, D., \& Rich, R. M. 2005, ApJ, 618, L129

Kalirai, J. S., Bergeron, P., Hansen, B. M. S., et al. 2007, ApJ, 671, 748

Kroupa, P. 2001, MNRAS, 322, 231

Lind, K., Charbonnel, C., Decressin, T., et al. 2011, A\&A, 527, A148

Marigo, P., Girardi, L., Chiosi, C., \& Wood, P. R. 2001, A\&A, 371, 152

Meng, X., Chen, X., \& Han, Z. 2008, A\&A, 487, 625

Milone, A. P., Bedin, L. R., Piotto, G., et al. 2008, ApJ, 673, 241

Milone, A. P., Piotto, G., Bedin, L. R., et al. 2010, in Proc. SF2A 2010, ed. S. Boissier, M. Heydari-Malayeri, R. Samadi, \& D. Valls-Gabaud, 319

Origlia, L., Valenti, E., Rich, R. M., \& Ferraro, F. R. 2006, ApJ, 646, 499

Piotto, G., Bedin, L. R., Anderson, J., et al. 2007, ApJ, 661, L53

Prada Moroni, P. G., \& Straniero, O. 2007, A\&A, 466, 1043

Prada Moroni, P. G., \& Straniero, O. 2009, A\&A, 507, 1575

Press, W. H., Flannery, B. P., \& Teukolsky, S. A. 1986, Numerical Recipes: The art of scientific computing (Cambridge: Cambridge University Press)

Renedo, I., Althaus, L. G., Miller Bertolami, M. M., et al. 2010, ApJ, 717, 183

Richer, H. B., Fahlman, G. G., Rosvick, J., \& Ibata, R. 1998, ApJ, 504, L91

Richer, H. B., Dotter, A., Hurley, J., et al. 2008, AJ, 135, 2141

Rohrmann, R. D., Althaus, L. G., \& Kepler, S. O. 2011, MNRAS, 411, 781

Rubin, K. H. R., Williams, K. A., Bolte, M., \& Koester, D. 2008, AJ, 135, 2163

Salaris, M., Cassisi, S., Pietrinferni, A., Kowalski, P. M., \& Isern, J. 2010, ApJ, 716,1241

Segretain, L., Chabrier, G., Hernanz, M., et al. 1994, ApJ, 434, 641

Torres, S., García-Berro, E., Burkert, A., \& Isern, J. 2002, MNRAS, 336, 971

Tremblay, P.-E., \& Bergeron, P. 2008, ApJ, 672, 1144

Twarog, B. A., Carraro, G., \& Anthony-Twarog, B. J. 2011, ApJ, 727, L7

van Loon, J. T., Boyer, M. L., \& McDonald, I. 2008, ApJ, 680, L49

Weiss, A., \& Ferguson, J. W. 2009, A\&A, 508, 1343

Weiss, A., Cassisi, S., Schlattl, H., \& Salaris, M. 2000, ApJ, 533, 413

Winget, D. E., Hansen, C. J., Liebert, J., et al. 1987, ApJ, 315, L77 